\title{
AID-ME: Automatic Identification of Dressing failures through Monitoring of patients and activity Evaluation
}

\author{
Aleksandar Matic ${ }^{\S}$, Priyal Mehta ${ }^{\ddagger}$, James M. Rehg $^{\ddagger}$, Venet Osmani $^{\S}$, Oscar Mayora ${ }^{\S}$ \\ \{priyal, rehg\}@cc.gatech.edu \{aleksandar.matic, venet.osmani, oscar.mayora\}@create-net.org \\ ${ }^{\S}$ Ubiquitous Interaction Group, CREATE-NET, via alla Cascata 56/D, Povo, Trento, Italy \\ ${ }^{\ddagger}$ Health Systems Institute and GVU Center, School of Interactive Computing, Georgia Institute of Technology, Atlanta, GA
}

\begin{abstract}
Monitoring and evaluation of Activities of Daily Living in general, and dressing activity in particular, is an important indicator in the evaluation of the overall cognitive state of patients. In addition, the effectiveness of therapy in patients with motor impairments caused by a stroke, for example, can be measured through long-term monitoring of dressing activity. However, monitoring of dressing activity has not received significant attention. In this paper, we describe a system that can automatically monitor dressing activity and identify dressing failures exhibited by patients. The system uses a synergistic combination of RFID and computer vision in order to identify a number of common dressing failures exhibited by the patients.
\end{abstract}

\section{INTRODUCTION}

Demographic changes in the developed world and current trends in the developing world are pointing towards a future where the number of elderly will increase significantly. These trends are occurring due to medical advances that have had the effect of prolonging the life of general population, which in turn has resulted in a significant shift of the median age of population. In this respect the increase in the number of elderly has also increased the need for care provision resources, thus exerting increasing strain on health care providers (the prevalence of dementia is doubling every five years in patients over the age of 65 [21]). The diseases that increasingly affect this population group are often associated with impairment of cognitive abilities and increased difficulty in performing the Activities of Daily Living (ADL).

ADL is a term that incorporates the basic tasks of everyday life such as eating, bathing, toileting, dressing and other common every-day activities [19]. There are a number of causes that affect the ability to perform ADL, thus impairing the independence of an individual's life. One of the most common causes is aging. According to the statistics of AoA (Administration of Aging) [1], by 2030 the number of older persons over 65 years is projected to be around 71.5 million only in the US. This is an increase of more than twice in comparison to the year 2000. Also, it is estimated that half of the elderly have some cognitive problems, while half of the elderly over the age of 85 exhibit symptoms that characterize Alzheimer's disease to varying degrees [2]. However, ADL impairments are not necessarily the consequence of an old age;

Permission to make digital or hard copies of all or part of this work for personal or classroom use is

granted without fee provided that copies are not made or distributed for profit or commercial advantage

and that copies bear this notice and the full citation on the first page. To copy otherwise, to republish,

to post on servers or to redistribute to lists, requires prior specific permission and/or a fee.

PERVASIVEHEALTH 2010, March 22-25, Munchen, Germany

Copyright @ 2010 ICST 978-963-9799-89-9

DOI 10.4108/ICST.PERVASIVEHEALTH2010.8895 diseases such as stroke or accident-related paralysis affect people of any age, impairing their motor skills and consequently affecting ADL. Therefore, it is important to monitor the performance of ADL of affected persons after an event that has resulted in such impairment. For example, the nursing homes that receive Medicare funds in the US must record and report the ADL performance of patients [3]. Due to the increasing prevalence of the diseases that affect ADL, healthcare institutions are struggling with the increasing care provisioning demand and shortage of specialists and caregivers. Adding to these costs is the requirement for long-term monitoring of patients in order to adjust the course of therapy, follow the progression of disease, or investigate the speed of recovery (e.g. after stroke [4]).

These issues have prompted healthcare institutions to investigate various cost-cutting strategies. In this respect, one promising direction is the development of smart environments to monitor ADL performance through technological solutions. Significant results have been obtained in the development of smart environments for monitoring hand washing [7, 15], cooking [13], and taking medications [16]. However, the task of monitoring dressing progress and failures, which is a basic daily activity, has not received the attention that it deserves. Walker et al [4] argue that dressing is a complex skill, important for the successful rehabilitation of stroke patients, and that it should be monitored continuously for many years. This is because there is a correlation between cognitive factors and dressing ability that can provide valuable information about the state of a patient's cognition. Moreover, the assessment of variations in patterns of daily activities, including dressing of elderly, and capturing patterns of change over time, are very important since they can be indicative symptoms of early dementia or can be used to measure the progress of the disease [5].

Typical practices in monitoring dressing activities involve therapists periodically taking notes while the patient performs the dressing steps $[11,12]$. This has three considerable disadvantages: (i) dressing is a very personal and private activity and carrying it out in front of another person is often uncomfortable and unpleasant; (ii) note taking is not only error prone (due to the tiredness of the therapist, for example), it is also subjective, creating difficulties in comparing the notes 
when different therapists assist the same patient; (iii) the presence of therapists can result in inconsistencies between the recorded performance of the activity and performance of the same activity carried out in the patients' usual environment, such as their home. This is because patients and especially the elderly will invest extra effort to carry out the activity correctly, and thus vindicate their independence, as was demonstrated in a study by Brown et al. [6].

In response to these issues, we have addressed the challenges in monitoring the dressing task, thereby enabling support for long-term assessment, through the use of two different technologies, namely computer vision and Radio Frequency Identification (RFID). In our design, the two technologies act in a complimentary manner, addressing the challenge of monitoring different aspects of a dressing task. The challenge relates to the fact that the set of dressing failures exhibited by patients cannot be reliably recognized with a single technology working in isolation. The set of most common failures relate to (i) putting clothes in an incorrect order (such as t-shirt over a jumper) [22], (ii) putting on clothes partially (such as only one sleeve is in) [4], (iii) putting on clothes backwards (inner part of a garment is on the outside) [23], and (iv) putting on too many layers of clothing and not adjusting garments with the temperature [22]. In conjunction with identifying dressing failures, it is also important to recognize when the correct dressing is performed as well.

A key requirement for a dressing monitoring system is to be unobtrusive, especially with regards to visible and wearable sensors (such as wrist worn devices). This is because wearable devices may influence the dressing activity and also because patients with dementia may tend to remove foreign objects. Therefore, in our system we have aimed to fulfil the requirements for an unobtrusive system, while achieving a high recognition rate both for correct dressing events and dressing failures.

The paper is organized as follows. Section II reviews the related work in the domain of smart environments intended for monitoring ADL. Our approach and description of the experiments are provided in Section III and IV respectively. Finally, we discuss the future work and draw the main conclusions.

\section{RELATED WORK}

The performance of a patient in the dressing task is often studied in medicine. Walker et al [4] investigate how dressing is affected during the rehabilitation after a stroke. They emphasize the types of failures as important parameters for determining relations between the nature of cognitive impairment and dressing ability. Dressing performance is analyzed very often in patients that suffer from Alzheimer's disease or other types of dementia (e.g. [11, 12]). Feyereisen et al [11] claim that there is both clinical and theoretical support for the importance of studying Alzheimer's patients' ability to perform the dressing task, since it is cognitively demanding and organised along a hierarchical plan with some repetitions of the same movement. They confirm that the impairments of dressing are quite common in patients suffering from dementia and they identify critical steps in the dressing task and perform a qualitative analysis of failures depending on the level of disease. Sometimes it is possible to restore dressing independence [20] for Alzheimer's patients, which also underlines the importance of monitoring that task. These works, as well as current practices, still rely on therapists to evaluate the patient's performance in person or sometimes subjects are recorded and video is analyzed by specialists (e.g. in [4]).

Numerous previous works have addressed the goal of monitoring ADLs in order to assess the subject's performance or to assist them in accomplishing tasks properly.

Cooking, as one of the most common household tasks, is addressed in [13]: This project provides assistance during the cooking process by showing snapshots of recent actions on a display positioned in the kitchen. The system acts as a memory aid and was shown to be helpful when subjects with memory problems were interrupted while performing the task. Wu et al [14] presented an approach to activity recognition based on object use that minimizes the amount of human-labelled data required for modelling. The combination of vision and RFID technologies is tested in a kitchen scenario and it achieves a recognition rate of $80 \%$ in 16 activities with 33 objects. The system described in [15] addresses the problem of recognizing steps in hand washing using video processing, with the main goal of assistance. Applying an HMM-based approach, an activity recognition rate of $79 \%$ is achieved. In their work on MedTracker [16], Hayes et al. developed an electronic pillbox that continuously monitors the medication-taking process over a certain period.

Previous research in ADL has primarily focused on inferring which activity is carried out at any given time. It has not addressed the evaluation of the performance of an activity.

One of the turning points in the field of inferring ADL is the project described in [3]. It addresses the recognition of 14 every-day activities based on the objects used, employing an RFID reader embedded in a glove while the characteristic objects for these tasks are tagged. The system reports not only the most probable activity but also the quality of performance to a certain extent, such as if a subject is wearing the same clothes each day. Dalton et al [18] evaluate the accuracy of ADL identification using wireless kinematic sensors depending on their position and on the manner of data processing. For the case of placing sensors on the ankle and hip, recognition rate is $81.2 \%$. The authors report dressing among the recognized activities. Using SVM and the data acquired from infrared sensors, microphones, door contact sensors, webcams and accelerometers, the design [17] also recognizes when a subject performs dressing/undressing in addition to 6 other daily activities. Ten ADLs are recognized in [19] using the fusion of RFID and accelerometers.

In the current literature there are no systems capable of monitoring the dressing task in detail, with the aim of distinguishing between the failure modes of this activity and correct dressing. However, there have been several papers in computer vision which concentrate on the problem of person detection. The work described in [20], for example, addresses the problem of estimating body shapes underneath clothing. The authors in [21] study the feature sets required for robust 
visual object detection, with human detection as a test case. In [22], the authors try to estimate the shape of clothing from images of the clothing. A network of cameras is used to analyze the fit of clothing on a person in [23]. The work presented in [24] is interesting in that it describes a method for segmenting clothes in an image using a set of examples. This method could be used to improve the results of segmentation in our work. In contrast to the aforementioned projects, our system provides the evaluation of the dressing task and it is capable of recognizing dressing failures along with correct dressing. In addition, dressing activities are recognized in an unobtrusive manner without affecting the natural course of the activity. Patients are not required to wear any heavy sensors and the technology is hidden away in the background.

\section{OUR APPROACH}

The aim of our project is to non-intrusively recognise the steps involved in a dressing task and evaluate the correctness of dressing activity. Once the relevant parameters in the task are recognized, the data is stored for each subject such that the changes in behaviour performance can be followed over extended periods of time in order to monitor the progress of disease (such as Alzheimer's disease) or the effectiveness of therapy and rehabilitation (in case of stroke or paralysis). The most common dressing failures that occur with patients are described below:

Putting on clothes in the wrong order - Patients with dementia are often confused with regard to the kind of garments that they are wearing; and thus put them in the wrong order, such as t-shirt over a jumper or a jacket (Figure 1a).

Putting on clothes backwards - Another common failure in a dressing task is putting garments on backwards, where the inner part of the garment is on the outside. This case often arises when patients take off their clothes and try to put them back on again (Figure 1b).

Putting on clothes only partially - Stroke patients, in particular, have difficulties with putting the clothes on properly. The failure is exhibited through failing to put the paretic hand through the correct sleeve. In that way, they end up with a garment only partially worn (Figure 1c).

The number of layers of clothing is not appropriate for the temperature - Judgement of warm and cold weather is impaired in dementia patients. As a consequence, these patients may put on too many or too few layers of clothing (Figure $1 \mathrm{~d}-$ e.g. indoor temperature).

The above failures are visually illustrated by experimental examples shown in Figure 1.

In order to detect the aforementioned failures and to monitor the dressing activity, we use information from both vision and RFID tags. Vision or RFID working in isolation can be used to detect specific types of failures. However, the two technologies separately have proven to be insufficient to recognize all of the failures reliably. Therefore, in order to reliably detect when a dressing failure has occurred, we fuse the information from vision with information coming from
RFID tags in order to detect whether a failure has occurred and identify the type of failure.
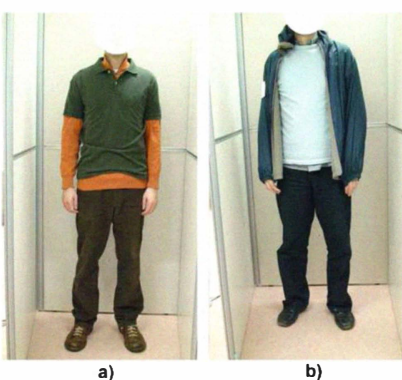

b)
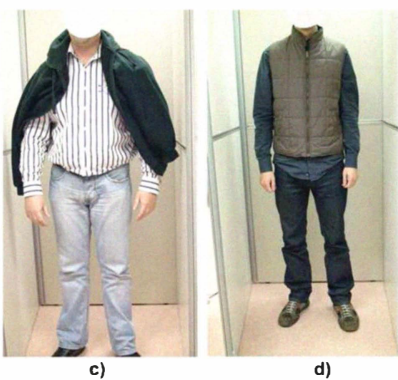

Figure 1 Common failures in dressing activity
The paragraphs that follow describe how each of the technologies contributes to the overall dressing failure identification system and the challenges we faced. Finally, a description of the fusion of the proposed technologies will be given.

\section{A. Experimental Setup}

Our setup is illustrated in Figure 2. The dressing booth had dimensions of $1 \mathrm{~m}$ by $1 \mathrm{~m}$ and in our experiments its size was large enough to allow ample space for all the manoeuvres necessary to perform dressing in a natural manner, as evidenced by the subjects that took part in the experiments. Two RFID antennas are positioned on each of the side walls and one on the back wall, $140 \mathrm{~cm}$ and $90 \mathrm{~cm}$ from the ground, respectively. A video camera positioned at the entrance of the dressing booth recorded image sequences as the dressing activity was taking place. The subject's face was automatically blurred, to address privacy concerns.

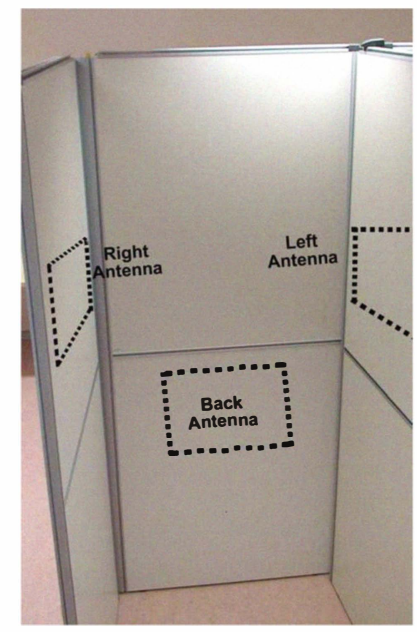

Figure 2 Dressing booth denoting the position of hidden RFID antennas

Upper garments are tagged inside on the shoulders and on the lower part of the back. We chose the lower part of the back for two reasons; first, to avoid possible interference between adjacent antennas and second, to increase the recognition rate of correct dressing since the backs of garments cannot be checked by the vision system, due to the camera position. 
Lower garments, such as pants were not tagged. We next provide a description of the RFID and vision setups.

\section{B. Monitoring of Dressing Activity with RFID}

Given the wide variety of RFID tags, which come in a wide range of shapes and sizes, we could easily identify ones that are small, non-intrusive, and almost invisible to our subjects (Figure 3). In our experiments, we have used an RFID system that works on $13.56 \mathrm{MHz}$ with passive tags (Figure 3) and antennas with a reading range of approximately $30 \mathrm{~cm}$. RFID antennas and associated hardware were hidden behind the dressing booth, thus our setup was free of observable sensors to allow as natural behaviour as possible.

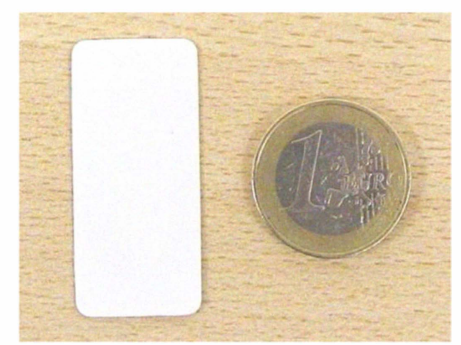

\section{Figure 3 RFID tags used in our experiments}

While some patients will dress correctly, others attempting the dressing activity will exhibit a number of failures. Individual failure categories along with the correct dressing, are described below:

\section{a) Putting on a garment correctly}

The mechanism of the recognition of dressing steps is based on detecting the tags attached to the shoulders and the back, once they are in the range of corresponding antennas (see Figure 2). When a person is facing the entrance of the dressing booth and a garment is put on properly, the right shoulder tag should be detected on the right antenna, the left shoulder tag on the left antenna, and the back tag on the back antenna. Furthermore, the back tag and only one shoulder tag are enough to detect that the garrnent is put on properly. However, as expected, we found from the experiments that tagging both shoulders instead of one improves the probabilities of detection of correct dressing.

\section{b) Getting the order of garments wrong}

Once we recognise that the garment is put on, it becomes easy to follow the sequence of events to determine the order in which the garments are put on and to infer an eventual failure in the ordering, such as putting a t-shirt over a jumper.

\section{c) Not adjusting clothes to weather conditions}

At the end of the task, the system is aware of which garments have been put on, which in conjunction with the information of weather conditions and simple reasoning, can provide appropriate recommendations if the number of layers is not adjusted properly.

\section{d) Putting on garments backwards}

Such event is recognized via the RFID system in similar manner to the recognition of correct dressing: the back tag is recognized with back antenna, left shoulder tag with right antenna and right shoulder tag with left antenna when the subject is facing the entrance of the dressing cabin.

\section{Bayesian Network Model}

In order to infer the dressing steps from the RFID readings, we developed a simple state model, shown in Figure 4. Nodes "Left", "Back" and "Right" correspond to the RFID antennas as positioned in the dressing cabin. "Left" and "Right" nodes can take "Left tag", "Right Tag" and "Not Detected" states while the node "Back" takes two states, "Back Tag Detected" or "Back Tag Not Detected". This means that the side antennas ignore the readings of a back tag and the back antenna ignores the readings of the shoulder tags. In this manner we can filter unintended readings that are not relevant to the dressing task, such as readings captured when a subject is moving inside the cabin or while the subject is holding a garment. "FinalState" node decides whether the garment is correctly put on, incorrectly put on or that the garment has not been put on. The probabilities of taking each of these states depend on the states of parental nodes and they are adjusted from training evidence.

Video image recordings provided the ground truth and were taken periodically from the beginning up to the end of the dressing task.

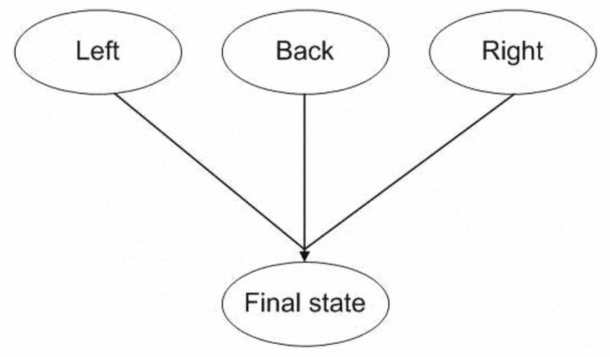

Figure 4 - Bayesian Network Model

Within the scope of our problem, the most significant limitation of RFID is that it cannot detect a dressing failure when a garment is put on partially (such as a shirt pulled on the neck only). This is due to the fact that our RFID antennas cannot compute the distance of the tag from the antenna, thus unable to spatially localise the tag within required granularity. Therefore, recognising the failure of partially worn clothes is identified with vision processing.

Using RFID technology introduces additional challenges in terms of tagging all of the garments with three tags. Depending on the number of garments, this might be a laborious task. However, it needs to be done only once because plastic RFID tags can be used which are water proof and therefore can be washed embedded in the clothes.

\section{Vision Processing to Identify Type of Failure}

In order to address the limitations of RFID, we use clustering of colors and their comparison to detect if a dressing failure has occurred. We take as input a pair of images, one from when the subject comes to the dressing booth in our lab 
and the other after the dressing activity has completed. As a pre-processing step, we perform clustering of each image on the basis of the colour of clothes and try to identify where the cluster lies spatially; whether it belongs to the top of the body or the bottom, or whether it corresponds to the background. Once this information is computed, we use a rule-based inference system that performs matching of the colour clusters, and their spatial positions, if needed, to identify if there is a dressing failure or not. The following paragraph explains the overall approach in more detail.

First, we perform background subtraction to isolate the person from the image. This is done by simply averaging the background images and subtracting them from the image with the foreground. We provide an initial bounding box around the person manually, and weigh the values that lie within the box with a higher value than the values that lie outside it. Then, thresholding is performed to obtain a mask for the foreground image. All pixel values lying outside the mask area are made white. Next, we obtain a feature vector for input to the k-means clustering algorithm. The feature vector contains the RGB values of the colours and their weighed spatial coordinates. The number of clusters $\mathrm{k}$ is incrementally checked to see if it lies in 3 to 8 . This is done by summing up the distance of all points from their respective centroids and comparing these values at each iteration. When there is a drop in the difference of these 2 values below a certain threshold limit, we stop increasing the number of clusters. Once k-means algorithm is performed, we get an image that is labelled with the different cluster values corresponding to each pixel. This serves as input to the spatial analysis phase. We define a horizontal line through the middle of the image. The part above the line is considered to be the top and the part below is the bottom. In order to determine uniquely which cluster corresponds to what part, the following approach is used: (i) Clusters corresponding to the white colour are background clusters and (ii) Conflict between a cluster belonging to the top part or the bottom part is resolved on the basis of the count for that label value. If the count in one part for that label value is negligible as compared to the count in the other part, then it is assumed that cluster belongs to the other part with the higher count. Now that we know whether a particular cluster label corresponds to the top or the bottom of the image, we can easily separate the two. We store the count value of each cluster label as well as the part that it corresponds to. This serves as our pre-processing task before we can actually perform the comparison of cluster colours for both images (see Figure 5).

$$
K_{\text {match,2 }}^{\text {before }}=\arg \min _{k}\left\|C_{2}^{\text {before }}-C_{k}^{\text {after }}\right\|
$$

We match the values of the centroids from the before and after image on the basis of their Euclidean distances, as shown in equation (1). The points which have the closest distance between them are said to form a match. A series of empirical checks are then performed on the matched clusters and also with the unmatched clusters. The following list summarizes them.

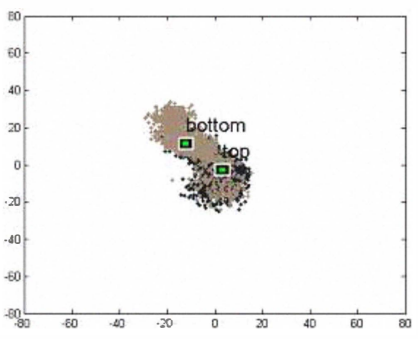

(a)

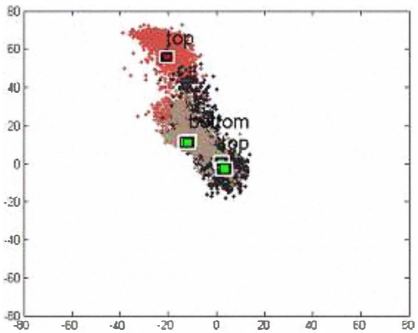

(c)

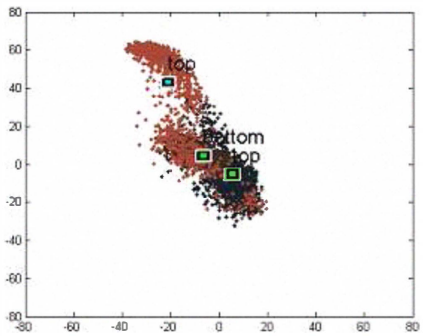

(e)

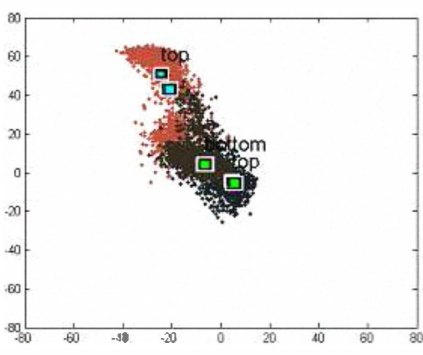

(g)

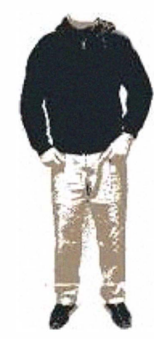

(b)

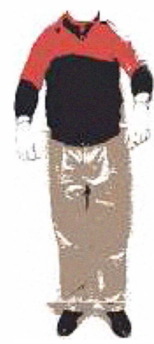

(d)

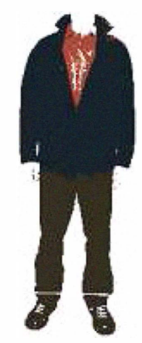

(f)

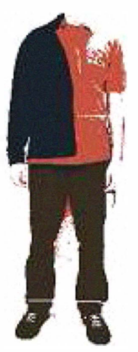

(h)
Figure 5- (a),(e) Plot showing colour clusters corresponding to before image (b),(f) Image labelled with cluster value and colour corresponding to centroids for before image (c),(g) Plot showing colour clusters corresponding to after image (d),(h) Image labelled with cluster value and colour corresponding to centroids for after image 


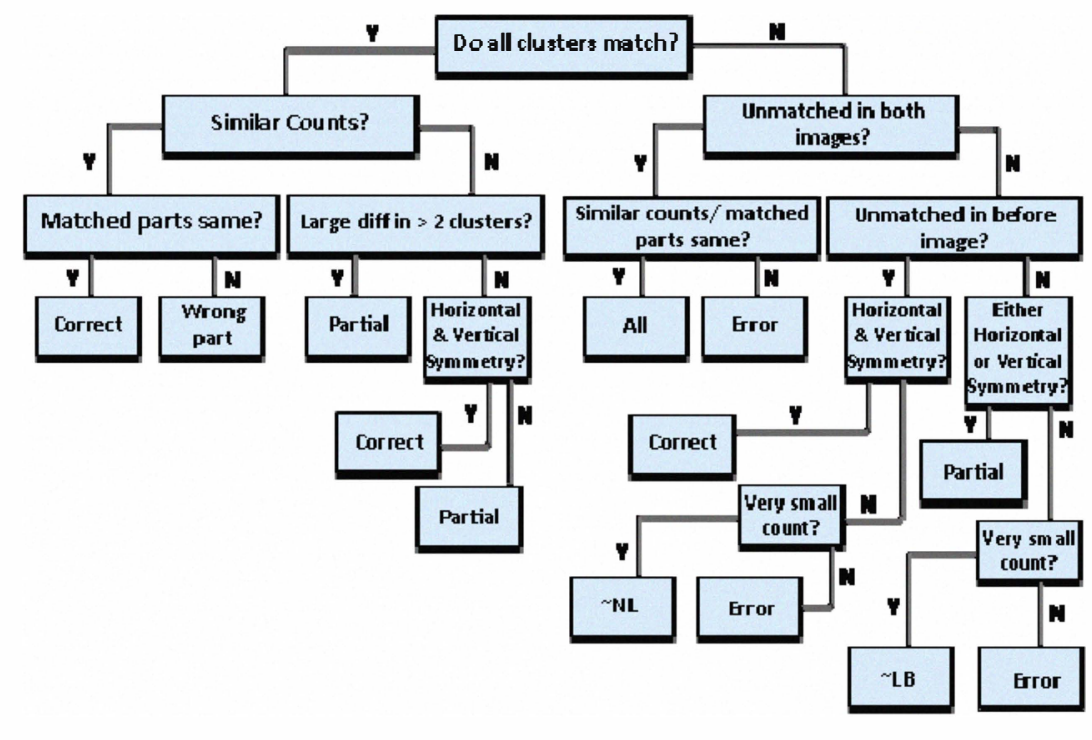

Figure 6 - Decision tree depicting inference system for cluster colour comparison

(i) For each group of pixels, if all the centroids match, we check if they have a similar count and whether they belong to the same part. If so, then we conclude that the dressing activity is correct.

(ii) For some groups of pixels, if their centroids match but their counts differ by a large amount, then this is classified as a partial dressing failure. A real world analogy to this would be wearing an unzipped jacket initially, so that you can see some parts of the colour of the t-shirt underneath but largely the jacket colour and then, wearing the jacket partially, as a result of which the t-shirt colour is more visible.

(iii) For some groups of pixels, if their centroids are unmatched in both the before and after cases, but they are similar in count and belong to the same part, we can say that one colour completely replaces another colour afterwards. This is possible in number of cases - if a new layer is worn on top of the already existing layer and it completely hides the one underneath it, or just the opposite- a layer, which completely hid the layer underneath, is not worn now. It is also possible that such layers of clothes are just worn out of order.

(iv) If the centroid for a group of pixels remains unmatched in the before image, we check to see if the number of pixels belonging to that group is much less. This would be the case when a scarf or some headgear which contributes a small amount of a different colour is worn initially but is not worn later on. This would also be the case when a shorter outerwear is worn on top of a longer innerwear in the earlier case and then later on the order of wearing them is reversed. Thus, we can conclude from these tests that a layer is either left behind or it is out of order, that is, a new layer has not been added. (v) In case the unmatched group of pixels from the earlier image has a sizable count, we check their spatial position. If the dominant colour that is matched in both the before and after images has horizontal and vertical symmetry, this can be classified as correct dressing. This case can be visualized as a person wearing a partly zipped jacket initially, as a result of which we can see a sizeable portion of the inner garment and then later on, he zips up the jacket completely and hence, hides the colour of the inner garment.

(vi) If there are unmatched clusters in the after image, we check spatially whether they have horizontal or vertical symmetry. If any of these checks fail, it is easy to conclude that a new garment is being worn but partially.

(vii) If, however, the count of the unmatched cluster dominates the count of the matched cluster from the same part, we can assume that a new layer has been added that partly hides the older layer or there is a change in the order of the clothes worn.

Figure 6 above illustrates the various steps the rule-based inference system takes in order to verify whether the processed images indicate a dressing failure or not. Due to the constraints on the vision system, it is sometimes not possible to exactly show that a failure is present; rather it is easier to show that a particular failure is not present in the input pair. As a result, the leaves of the decision tree in case of incorrect dressing say that the failure is 'Not a new layer' ( NL) or 'Not left behind a layer' $(\sim \mathrm{LB})$ or All, which indicates that either there could be a new layer or a layer could have been left behind or the layers are out of order. 


\section{EXPERIMENTAL RESULTS}

\section{A. RFID}

The RFID design was tested using a t-shirt and jumper tagged on both shoulders and back, winter jacket tagged on left shoulder and back and light jacket tagged on right shoulder and back. As expected, correct dressing of single garment was inferred $10-20 \%$ more reliably for three-tagged garments, namely a t-shirt and a jumper in comparison to the a light and a winter jackets that had only two tags.

We recruited 11 participants that were not connected to this research. They were asked to choose any combination of clothes they wanted and to perform the dressing task without further instructions; as they would do at home. Initially they performed correct dressing. Then, they were asked to perform two out of three failures (putting on the garments the inside out, in the wrong order or putting garments partially), which were described by the researchers. As noted previously, the RFID system was unable to address the failure of partially putting on a garment and as such was left for the fusion with vision processing. We collected 52 trials overall that were used for testing the fusion approach as well (described later). Although the side antennas were positioned at the height of $140 \mathrm{~cm}$ from the ground to detect shoulder tags and the back antenna at $90 \mathrm{~cm}$ height from the ground to detect back tag, the range was enough to capture the tags despite variations in participants' height (the tallest was $197 \mathrm{~cm}$ while the shortest was is $165 \mathrm{~cm}$ ).

Table 1 shows the results of experiments for the RFID system. The last column represents the situations in which the system reported "unrecognized event" when the subjects have put garments in a correct way, backwards or incorrect order. These false negatives were the consequence of missing two tags at the same time. On the other hand, detecting two or three tags on the corresponding antennas at the same moment is unlikely to happen when a garment is not put on (such as when a subject is holding a garment inside the dressing booth), which is confirmed by the absence of false positives in our experiments (last row in Table 1).

Table 1 - RFID results

\begin{tabular}{|c|c|c|c|c|}
\hline Event Type & $\begin{array}{c}\text { Correct } \\
\text { Dressing }\end{array}$ & $\begin{array}{c}\text { Wrong } \\
\text { Order }\end{array}$ & Backwards & Unrecognized \\
\hline $\begin{array}{c}\text { Correct } \\
\text { Dressing }\end{array}$ & $83.9 \%$ & $0 \%$ & $0 \%$ & $16.1 \%$ \\
\hline Wrong Order & $0 \%$ & $80 \%$ & $0 \%$ & $20 \%$ \\
\hline Backwards & $0 \%$ & $0 \%$ & $83.3 \%$ & $16.7 \%$ \\
\hline Unrecognized & $0 \%$ & $0 \%$ & $0 \%$ & - \\
\hline
\end{tabular}

\section{B. Vision}

The following table, Table 2 shows the confusion matrix generated when testing images for: (1) Correct Dressing (2) Partial Dressing Failure (3) Wrong Dressing. Table 3 is a breakdown of the different cases related to Wrong Dressing.
Table 2 - Vision results

\begin{tabular}{|c|c|c|c|}
\hline Event Type & $\begin{array}{c}\text { Correct } \\
\text { Dressing }\end{array}$ & $\begin{array}{c}\text { Partial } \\
\text { Dressing }\end{array}$ & $\begin{array}{c}\text { Wrong } \\
\text { Dressing }\end{array}$ \\
\hline $\begin{array}{c}\text { Correct } \\
\text { Dressing }\end{array}$ & $73 \%$ & $9 \%$ & $18 \%$ \\
\hline $\begin{array}{c}\text { Partial } \\
\text { Dressing }\end{array}$ & $0 \%$ & $100 \%$ & $0 \%$ \\
\hline $\begin{array}{c}\text { Wrong } \\
\text { Dressing }\end{array}$ & $14 \%$ & $0 \%$ & $86 \%$ \\
\hline
\end{tabular}

Table 3 - Wrong Dressing results

\begin{tabular}{|c|c|c|c|}
\hline Event Type & All & $\sim$ NL & $\sim$ LB \\
\hline All & $100 \%$ & $0 \%$ & $0 \%$ \\
\hline$\sim$ NL & $0 \%$ & $100 \%$ & $0 \%$ \\
\hline$\sim$ LB & $0 \%$ & $0 \%$ & $100 \%$ \\
\hline
\end{tabular}

We have used 30 image pairs for verifying our algorithm. The survey of images brings forth the following limitations in the method used. The background subtraction algorithm is very naïve, and can fail when the clothing is similar in colour to the background. It is also sometimes difficult to distinguish between clusters of 2 colours when they are proximal in value and spatial position. Moreover, garments with multiple colours or having complex patterns will make it difficult to perform matching and failure detection.

\section{Fusion of RFID and Vision Processing}

Table 4 presents the confusion matrix for the fusion of RFID and vision observations. Recognition of the case of putting garments on backwards can be done with RFID only, while the failure of putting on the garment partially can only be detected through vision processing. This is the motivation for the joint development and integration of the two systems. The results for correct dressing and wrong order detection further illustrate the complementary nature of the two systems. The fusion provides $10 \%$ higher recognition rate for inferring the wrong order of dressing in comparison to the RFID system working in isolation, where the overall improvement increases from $83.9 \%$ (RFID) and $73 \%$ (Vision) to $93.5 \%$.

Table 4 - Fusion Results

\begin{tabular}{|c|c|c|c|c|c|}
\hline Event Type & $\begin{array}{c}\text { Correct } \\
\text { Dressing }\end{array}$ & $\begin{array}{c}\text { Wrong } \\
\text { Order } \\
\text { Dressing }\end{array}$ & $\begin{array}{c}\text { Backwards } \\
\text { Dressing }\end{array}$ & $\begin{array}{c}\text { Partial } \\
\text { Dressing }\end{array}$ & $\begin{array}{c}\text { Un- } \\
\text { recognized }\end{array}$ \\
\hline $\begin{array}{c}\text { Correct } \\
\text { Dressing }\end{array}$ & $93.5 \%$ & $0 \%$ & $0 \%$ & $0 \%$ & $6.5 \%$ \\
\hline $\begin{array}{c}\text { Wrong Order } \\
\text { Dressing }\end{array}$ & $0 \%$ & $90 \%$ & $0 \%$ & $0 \%$ & $10 \%$ \\
\hline $\begin{array}{c}\text { Backwards } \\
\text { Dressing }\end{array}$ & $0 \%$ & $0 \%$ & $83.3 \%$ & $0 \%$ & $16.7 \%$ \\
\hline $\begin{array}{c}\text { Partial } \\
\text { Dressing }\end{array}$ & $0 \%$ & $0 \%$ & $0 \%$ & $100 \%$ & $0 \%$ \\
\hline Unrecognized & $0 \%$ & $0 \%$ & $0 \%$ & $0 \%$ & - \\
\hline
\end{tabular}




\section{CONCLUSION AND FUTURE WORK}

We have demonstrated that the fusion of RFID and vision processing systems can reliably detect a number of most common failures in dressing that patients with various impairments exhibit. Some of the failures can only be identified with one of the two systems, thus making the fusion a requirement, while for the failures that both systems were capable of recognizing, the experimental results showed an appreciable improvement in the recognition rate. As a result, we achieved a robust platform for monitoring dressing activity. The system provides a platform to provision assistance to patients about eventual failures, thus helping patients in one of the basic daily activities. In addition, it allows for the possibility of long term tracking of eventual changes in dressing performance, which could be used as indicators in the evaluation of the overall cognitive state.

\section{ACKNOWLEDGMENTS}

The research was funded by the Autonomous Province of Trento, Call for proposal Major Projects 2006 (project ACube).

\section{REFERENCES}

[1] Administration of Aging. www.aoa.gov, Last time accessed: November 2009.

[2] M. Morris, J. Lundell, E. Dishman, and B. Needham, New Perspectives on Ubiquitous Computing from Ethnographic Study of Elders with Cognitive Decline. UbiComp'03, 2003.

[3] M. Philipose, K. Fishkin, M. Perkowitz, D. Petterson, D. Fox, H. Kautz, D. Hanhel, Inferring Activities from Interactions with Objects. Context Aware Computing, 2004.

[4] C. M. Walker, A. Sunderland, J. Sharma, M. F. Walker, The impact of cognitive impairment on upper body dressing difficulties after stroke: a video analysis of patterns of recovery. J Neurol Neurosurg Psychiatry; 75:43-48, 2004

[5] J. Kaye, Home based technologies: A new paradigm for conducting dementia prevention trials. NIA - Layton Aging \& Alzheimer's Disease Center and ORCATECH, the Oregon Center for Aging \& Technology, Oregon Health \& Science University, Portland, USA, 2008.

[6] C. Brown, W.P. Moore, D. Hemman, and A. Yunek, Influence if instrumental activities of daily living assessment method on judgements of independence. American Journal of Occupational Therapy, Mar;50(3):pp:202-206, 1996.

[7] C. Peters, S. Wachsmuth, J. Hoey, Learning to recognise behaviours of persons with dementia using multiple cues in an HMM-based approach. Proceedings of the 2nd International Conference on Pervasive Technologies Related to Assistive Environments, 2009.

[8] Dressing Rehabilitation Evaluation Stroke Study, http://www.nottingham.ac.uk/ chs/ research/projects/dress.aspx, Last time accessed: November 2009.

[9] S. P. Mokashi, Relationship between cognitive deficits and the ability to perform the activities of daily living in stroke patients. The Indian Journal of Occupational Therapy : Vol XXXVII : No. 1, 2005.

[10] M. M. Rahman, H. S. Kim, S. Ishikawa, Solving a Dress Problem for a Human Model Recognition. Proceedings of the $40^{\text {th }}$ SICE Annual Conference, International Session Papers, Page(s): 210-213, 2001.
[11] P. Feyereisen, M. Gendron, and X. Seron, Disorders of Everyday Actions in Subjects Suffering from Senile Dementia of Alzheimer's Type: An Analysis of Dressing Performance. Neuropsychological Rehabilitation, 9(2), 169-188, 1999.

[12] K.. Namazi, Dressing independently: A closet modification model for Alzheimer's disease patients, American Journal of Alzheimer's Disease and Other Dementias. Vol. 7, No. 1, 22-28, 1992.

[13] E. D. Mynatt, A. S. Melenhorst, A. D. Fisk, W. A. Rogers, Aware Technologies for Aging in Place: Understanding User needs and Attitudes, Georgia Institute of Technology, IEEE Pervasive Computing, Pages 36-41, April-June 2004.

[14] J. Wu, A. Osuuntogun, T. Choudhury, M. Philipose, and J. M. Rehg, $A$ Scalable Approach to Activity Recognition based on Object Use. Computer Vision, 2007, ICCV 2007, IEEE $11^{\text {th }}$ International Conference on, Computer Vision, 2007.

[15] A. Mihailidis, B. Carmichael, and J. Boger, The Use of Computer Vision in an Intelligent Environment to Support Aging-in-Place, Safety, and Independence in the Home, IEEE Transactions on Informations Technology in Biomedicine, Vol. 8, No. 3, September 2004

[16] T. L. Hayes. J. M. Hunt, A. Adami, and J. A. Kaye, An Electronic Pillbox for Continuous Monitoring of Medication Adherence. Proceedings of the $28^{\text {th }}$ IEEE EMBS Annual International Conference, New York City, USA, 2006.

[17] A. Fleury, N. Noury, and M. Vacher, Supervised Classification of Activituies of Daily Living in Health Smart Homes using SVM. 31st Annual International Conference of the IEEE EMBS, Minneapolis, Minnesota, USA, 2009.

[18] A. F. Dalton, F. Morgan, and G. O. Laighin, A preliminary study of using wireless kinematic sensors to identify basic Activities of Daily Living. $30^{\text {th }}$ Annual International IEEE EMBS Conference, Vancouver, British Columbia, Canada, 2008.

[19] M. Stikic, T. Huynh, K. V. Laerhoven, and B. Schiele, ADL Recognition Based on the Combination of RFID and Accelerometer Sensing. PervasiveHealth 2008, Second International Conference on Pervasive Computing Technologies for Healthcare, 2008.

[20] A. O. Bãlan and M. J. Black, The Naked Truth:Estimating Body Shape Under Clothing.Proceedings of the $10^{\text {th }}$ European Conference on Computer Vision, 2008.

[21] N. Dalal and B. Triggs, Histograms of Oriented Gradients for Human Detection, International Conference on Computer Vision \& Pattern Recognition. Volume 2. 886-893, 2005

[22] A. Lobay, D. A. Forsyth, Recovering Shape and Irradiance Maps from rich dense texton fields, Proceedings of the IEEE Computer Society Conference on Computer Vision and Pattern Recognition, 2004.

[23] W. Zhang, J. Beggole, M. Chu,, J. J. Liu, N. Yee, Real-time clothes comparison based on multi-view vision. $2^{\text {nd }}$ ACM/IEEE International Conference on Distributed Smart Cameras, 2008.

[24] A. Gallagher, T. Chen, Clothing Cosegmentation for Recognizing People, IEEE Conference on Computer Vision and Pattern Recognition, 2008.

[25] K. K. Engelman, Restoring dressing independence in persons with Alzheimer's disease: A pilot study. American Journal of Alzheimer's Disease and Other Dementias, Vol. 17, No. 1, 37-43, 2002.

[26] J. Lindsay, E. Sykes, I. Mcdowell, R. Verreault, and D. Laurin, More than the epidemiology of Alzheimer's disease: Contributions o the Canadian study of health and aging, Can. J. Psychiatry, vol. 49, no. 2, pp 83-91, 2004.

[27] Alzheimer's Australia, An Australian Government Initiative, Dressing Caring for someone with dementia, Help Sheet, 2005.

[28] C. G. Lyketsos and P. V. Rabins, Dementia Care Guidelines for Families, Second Edition, Division of Geriatric Psychiatry and Neuropsychiatry The Johns Hopkins University, 2006. 